\title{
Testing olfactory dysfunction in acute and recovered COVID-19 patients: a single center study in Italy
}

\author{
Jacopo Pasquini ${ }^{1,2}$ (D) $\cdot$ Carlo Maremmani $^{3}$ (D) Stefano Salvadori $^{4}$ (D) Vincenzo Silani $^{1,2}$ (D) Nicola Ticozzi ${ }^{1,2}$
}

Received: 8 November 2020 / Accepted: 17 March 2021 / Published online: 26 March 2021

(C) The Author(s) 2021

\begin{abstract}
Background Olfactory dysfunction in coronavirus disease 2019 (COVID-19) is common during acute illness and appears to last longer than other symptoms. The aim of this study was to objectively investigate olfactory dysfunction in two cohorts of patients at two different stages: during acute illness and after a median recovery of 4 months.

Methods Twenty-five acutely ill patients and 26 recovered subjects were investigated. Acute patients had a molecular diagnosis of COVID-19; recovered subjects had a positive antibody assay and a negative molecular test. A 33-item psychophysical olfactory identification test tailored for the Italian population was performed.

Results Median time from symptoms onset to olfactory test was 33 days in acute patients and 122 days in recovered subjects. The former scored a significantly higher number of errors at psychophysical testing (median [IQR]: 8 [13] vs 3 [2], $p<0.001$ ) and were more frequently hyposmic $(64 \%$ vs $19 \%, p=0.002)$. Recovered subjects reported a variable time to subjective olfactory recovery, from days up to 4 months. Participants included in the study reported no significant nasal symptoms at olfactory testing. Among recovered subject who reported olfactory loss during acute COVID-19, four (27\%) were still hyposmic. Demographic and clinical characteristics did not show significant associations with olfactory dysfunction.

Conclusion Moderate-to-severe hospitalized patients showed a high level and frequency of olfactory dysfunction compared to recovered subjects. In the latter group, subjects who reported persisting olfactory dysfunction showed abnormal scores on psychophysical testing, indicating that, at least in some subjects, persistent hyposmia may represent a long-term sequela of COVID-19.
\end{abstract}

Keywords Anosmia $\cdot$ Hyposmia $\cdot$ Olfactory testing $\cdot$ COVID-19 $\cdot$ SARS-CoV-2

\section{Introduction}

Olfactory dysfunction in coronavirus disease 2019 (COVID19) is a common symptom appearing during the acute phase

Nicola Ticozzi

n.ticozzi@auxologico.it

1 Department of Neurology and Stroke Unit and Laboratory of Neuroscience, Istituto Auxologico Italiano IRCCS, Milan, Italy

2 Department of Pathophysiology and Transplantation, Dino Ferrari Center, Università degli Studi di Milano, Milan, Italy

3 Neurology Unit, Ospedale Apuane - Azienda USL Toscana-Nord Ovest, Massa, Italy

4 Institute of Clinical Physiology, National Research Council (CNR), Pisa, Italy of the disease [1-5]. The frequency and degree of olfactory dysfunction during COVID-19 was assessed in some studies through heterogeneous psychophysical olfactory tests, mostly in mild disease [6-10]. These reports have shown high prevalence of olfactory dysfunction, from 40 to $98 \%$. One study in hospitalized patients found that about 40 days after symptoms onset, olfactory dysfunction could be detected in $21 \%$ of patients [11]. Conversely, little is known about subjective and objective recovery of olfactory function in COVID-19, as only a few studies have been performed and their observation period was limited from 2 to 8 weeks after symptoms onset [12-15]. While these studies have shown relatively high recovery rates in the first weeks after symptoms onset, they still report around $40 \%$ of patients affected by olfactory dysfunction at the end of their follow-up. Overall, olfactory dysfunction in hospitalized patients with moderate-to-severe disease and long-term recovery of olfactory function after COVID-19 have not been well established. 
In this study we aimed to describe the features of COVID19-associated olfactory dysfunction in hospitalized patients with acute, moderate-severe COVID-19 as well as in recovered subjects whose symptoms onset was at least 3 months prior. Olfactory dysfunction was investigated through psychophysical testing that was carried out with a 33-item suprathreshold olfactory test for which normative values have previously been determined in a population of 511 healthy controls [16].

\section{Methods}

\section{Study design}

This cross-sectional, two-phase study was designed to evaluate and compare COVID-19 olfactory dysfunction in acutely ill patients and recovered subjects. A cohort of 25 COVID-19 moderate-severe patients was enrolled while hospitalized at our institution in April 2020; a second cohort of 26 COVID19 recovered subjects was enrolled between June and September 2020.

\section{Participants' selection}

The study was conducted at Istituto Auxologico Italiano IRCCS, Milan, Italy. Exclusion criteria for all participants were pre-existing olfactory or taste disturbances; invasive and non-invasive mechanical ventilation; oxygen therapy and inability to tolerate room air for less than $30 \mathrm{~min}$; ongoing acute or chronic sinusitis; past severe head trauma; Parkinson disease, Alzheimer disease or other types of dementia; and inability to communicate verbally with the clinician (e.g., language barrier).

The cohort of 25 acute COVID-19 patients was selected among 54 patients who were present at our institution between 20th April and 30th April 2020. All patients had a diagnosis of COVID-19 by SARS-CoV-2 RNA nucleic acid amplification test (NAAT) on naso-oropharyngeal swab.

Twenty-six recovered subjects were selected among employees of Istituto Auxologico Italiano and close relatives who a positive COVID-19 antibody assay that was carried out as a screening program. All tested negative at a subsequent SARS-CoV-2 RNA NAAT. Eight of these subjects had a molecular diagnosis through NAAT during acute disease. Serological testing was carried out with either one of two commercially available kits (Diasorin Liaison ${ }^{\circledR}$ SARSCOV-2 S1/S2, Roche Elecsys ${ }^{\circledR}$ Anti-SARS-CoV-2).

Before olfactory testing, all participants underwent a structured interview regarding olfactory or gustatory disturbances before and during the disease, and the total nasal symptom score questionnaire (3 items, total score 0-9) [17] (Supplementary Material).

\section{Psychophysical olfactory testing}

All participants were tested without respiratory personal protective equipment (e.g., surgical mask). Psychophysical olfactory testing was performed with the Italian Olfactory Identification Test (IOIT), which includes 33 odorants, each one absorbed into a small, rectangular, white cardboard testing card contained inside a sealed aluminum envelope. All components of the test, i.e., 33 small aluminum envelopes each containing a testing card, are for single-use and disposable. Each envelope is numbered and matched with the corresponding multiple-choice question on the answer sheet; each multiple-choice question is composed of four possibilities, and only one is the correct answer. Once an aluminum envelope is opened, the odor is released and the patient is asked "Do you smell something? If so, what smell do you detect?." The patient has to pick an answer even in the case no odor is detected. The 33 odorants are commonly present in the Italian culture and cover a wide range: citrus, floral, sweet, woodlike, minty, and unpleasant (full list in Supplementary Material). The upper reference limits of errors, above which the test is considered abnormal and the subject hyposmic, are derived from an Italian cohort of 511 healthy volunteers. Normative values have been previously computed using mean +2 standard deviations; the 97.5 percentile; and $95 \%$ individual confidence curves of a third-order polynomial regression. They are the same for males and females: 4 errors below age 49, 5 errors between 50 and 59, 6 errors between 60 and 69,7 errors over 70 years of age [16].

\section{Statistical analysis}

Descriptive statistics are reported as numbers and percentages, or medians and interquartile ranges (IQRs). Comparisons between two groups were performed with non-parametric tests. In order to compare the central values of a quantitative variable (e.g., number of errors at psychophysical testing) between two groups, Mann-Whitney $U$-test was performed. The comparison of the distribution of a continuous variable (e.g., age) across two groups was performed with the Kolmogorov-Smirnov twosample test. In order to test the relationship between two categorical variables (e.g., sex across two groups) the Fisher's exact test was used, while for two continuous or ordinal variables Spearman rank test was used. The significance threshold for hypothesis testing was $p<0.05$. Statistical analysis was carried out with Statistical Package for Social Sciences (IBM® SPSS) version 21.

\section{Results}

Fifty-one subjects were included in the study: 25 acute COVID19 hospitalized patients and 26 recovered subjects. Median 
(IQR) age was 52.1 (24.1) years and 27 subjects (53\%) were female. Recovered subjects were significantly younger than hospitalized patients $(U=59.0, p<0.001)$. Sex across the two groups was evenly distributed $\left(\chi^{2}=3.296, p=0.095\right)$. Demographic and general clinical characteristic are shown in Table 1.

No subject included in the study reported olfactory loss as the first isolated symptom. Four patients in each cohort reported the onset of olfactory symptoms to be concomitant with other COVID-19 symptoms.

Median time (IQR) from first symptoms to psychophysical olfactory testing was 33 (9) days for hospitalized patients and 122 (51) days for recovered subjects; the latter group was tested at least 90 days after symptoms onset. At the time of testing, no participant showed rhinorrhea, nasal obstruction, or other nasal symptoms; all had a TNSS of zero or one.

The number of errors at psychophysical olfactory testing was significantly higher in the hospitalized group compared to the recovered group (median [IQR]: 8 [13] vs 3 [2], $U=114.0$, $p<0.001$ ). Based on psychophysical olfactory test normative values, the proportion of hyposmic subjects was significantly higher in hospitalized patients compared to recovered subjects (64\% and 19\%, respectively, $\chi^{2}=10.546, p=0.002$; Table 2).

\section{Olfactory and clinical characteristics of hospitalized patients}

Eight patients (32\%; four females) experienced subjective olfactory disturbances during the disease, and of these, seven noted also gustatory disturbances. Three (12\%) of those eight patients reported persisting subjective olfactory disturbances at the time of psychophysical testing, while five declared a subjective recovery of olfactory function. Interestingly, abnormal IOIT scores were observed in all patients who complained of subjective hyposmia at the time of testing, but also in thirteen individuals (52\%) unaware of their deficit. These results are summarized in Table 3. Overall, four of ten females and twelve of 15 males (total: 16 of $25,64 \%$ ) showed olfactory dysfunction $\left(\chi^{2}=4.167, p\right.$-value $\left.=0.087\right)$. Age distribution in hyposmic and normosmic patients did not differ significantly (median age [1st-3rd quartiles] 68 [59-77] and 58 [49-67]; $d$ $=0.883, p$-value $=0.416$ ).
Eighteen patients $(72 \%)$ needed some kind of respiratory support during the disease (all oxygen therapy; twelve noninvasive mechanical ventilation and of these, three needed invasive mechanical ventilation; all were tested once they could tolerate room air for at least $30 \mathrm{~min}$ ). The presence of olfactory dysfunction and the number of errors at olfactory testing were not associated with age, disease duration, and the need for mechanical ventilation (Supplementary Table 1). Two patients died due to in-hospital complications (one pneumomediastinum, one multi-organ failure). The remaining patients were discharged from the COVID unit. These 23 patients underwent a followup telephone interview in February 2021, in order to assess their subjective level of smell: five previously normosmic patients did not report any smell complaint; three patients with COVID-19 hyposmia reported a partial recovery; six patients with either subjective smell loss or abnormal olfactory test score reported a complete recovery; and nine patients were lost to follow-up (one died, eight could not be reached by telephone).

\section{Olfactory and clinical characteristics of recovered subjects}

All subjects in the recovered group had evidence of prior COVID-19 infection detected through antibody testing and a concomitant negative SARS-CoV-2 RNA NAAT on nasopharyngeal swab. Five subjects did not have a history of COVID-19 symptoms. Eight subjects had a molecular diagnosis of COVID-19 during the acute phase through NAAT.

Fifteen subjects $(58 \%)$ reported subjective olfactory disturbances (anosmia or severe hyposmia) during acute COVID-19; eleven of these also complained gustatory problems. Two patients reported only gustatory disturbances. At the time of olfactory testing, eleven of 15 subjects reported a complete recovery. At psychophysical testing, a total of five subjects (19\%, three females and two males) were hyposmic, while twenty subjects (77\%, including all asymptomatic subjects) who described a subjectively normal olfactory function had normal test scores; one subject who complained persistent hyposmia following SARS-CoV-2 symptomatic infection had a normal test score (Table 4). Subjective median time to recovery in eleven subjects who reported full recovery of olfactory function was 45 days (IQR: 43; range: 9-100; Table 2).
Table 1 Demographic and general clinical characteristics of the 51 participants enrolled in the study

\begin{tabular}{lll}
\hline & Hospitalized patients & Recovered subjects \\
\hline Number & 25 & 26 \\
Sex, M/F & $15 / 10$ & $9 / 17$ \\
Age, median (IQR) & $66.7(19.1)$ & $46.4(12.5)$ \\
Asymptomatic/symptomatic for COVID-19 ${ }^{1}, n$ & $0 / 25$ & $5 / 21$ \\
Interstitial pneumonia at chest imaging (X-ray or CT scan), $n$ & $23(92 \%)$ & $2(8 \%)$ \\
\hline
\end{tabular}

${ }^{1}$ Symptoms included: fever, cough, dyspnea, diarrhea, nasal congestion, rhinorrhea, olfactory and taste disturbances COVID-19, coronavirus disease 2019; CT, computed tomography 
Table 2 Summary of characteristic of olfactory disturbances in the two groups included in the study

\begin{tabular}{|c|c|c|c|}
\hline & Hospitalized patients $(n=25)$ & Recovered subjects $(n=26)$ & $p$ \\
\hline Subjective olfactory or gustatory disturbances during COVID- $19, n(\%)$ & $10(40 \%)$ & $15(57 \%)$ & - \\
\hline $\begin{array}{l}\text { Subjective olfactory or gustatory disturbances at olfactory test administration, } \\
n(\%)\end{array}$ & $3(12 \%)$ & $4(15 \%)$ & - \\
\hline Time from symptoms onset to olfactory testing, median days (IQR), range & $33(12), 9-51$ & $122(51), 91-171$ & - \\
\hline Number of errors at psychophysical olfactory test, median (IQR), range & $8(13), 1-24$ & $3(2), 0-13$ & $<0.001^{1}$ \\
\hline Hyposmia at psychophysical olfactory test, $n(\%)$ & $16(64 \%)$ & $5(19 \%)$ & $0.002^{2}$ \\
\hline Hyposmic subjects, M/F, number (percentages ${ }^{3}$ ) & $12 / 4(80 \% / 40 \%)$ & $2 / 3(22 \% / 18 \%)$ & \\
\hline
\end{tabular}

No relationship was found between the number of errors at psychophysical testing and the number of days since COVID19 symptoms onset ( rho $=-0.166, p=0.472$ ). The number of errors at olfactory testing was associated with age (rho = $0.530, p=0.05$ ) but the distribution of age in subjects with and without hyposmia was not significantly different (median age [1st-3rd quartiles] 49 [47-54] and 42 [36-48], respectively; $d=1.244, p$-value $=0.091$ ).

\section{Discussion}

In this study we investigated COVID-19-related olfactory dysfunction through clinical interviews and psychophysical testing. We carried out a cross-sectional, comparative study in moderate-severe COVID-19 hospitalized patients and subjects who had recovered from prior SARS-CoV-2 infection. Psychophysical testing was performed with a 33 odor identification test previously validated in a population of 511 Italian healthy volunteers [16].

While recent evidence based on psychophysical testing has shown a high prevalence of olfactory dysfunction in the acute phase of the disease $[6,8,10]$, little is known about long-term olfactory function recovery. Indeed, only a few studies assessed olfactory function using psychophysical tests, and all were limited to maximum 8 weeks after symptoms onset $[12,13,15]$. In our analysis, all recovered subjects were tested at least 90 days after symptoms onset and more than half after 4 months.

Awareness of olfactory dysfunction was low in the hospitalized group and high in the recovered group. In the latter, only three of 26 patients had conflicting results between subjective perception and psychophysical test. In hospitalized patients, low awareness of olfactory dysfunction may have been shadowed by the presence of more troubling symptoms, hospitalization, and respiratory support techniques. Data of the hospitalized group, in which $64 \%$ of patients were hyposmic, were collected during the early phase of the epidemic in Northern Italy and are in agreement with other studies carried out in Italy [10] and elsewhere [6-8]. Varying frequencies of olfactory dysfunction are likely related to psychophysical testing heterogeneity and different time intervals from symptoms onset to testing. We tested our patients a median of 33 days after symptoms onset: those who were tested later in the disease course needed non-invasive or invasive mechanical ventilation, thus could not be tested earlier.

A few recent prospective studies investigated the reversibility of olfactory dysfunction in COVID-19 [12, 13]. In a hospitalbased study in Iran, patients were tested twice with the country's version of the UPSIT at an interval of 1 or 4 weeks. They showed that $60 \%$ of subjects recovered normosmia over 7 to 8 weeks after symptoms onset. Another study in Italy [13] showed that $67 \%$ of patients had olfactory dysfunction at symptoms onset, and it was still present in $56 \%$ after 20 days. These studies concluded that a high proportion of patients recovered olfactory function in a short time interval; however, many patients still had a detectable olfactory dysfunction. In our study, 19\% of subjects presented olfactory dysfunction after a median followup of 4 months. It must be recognized that three of the five hyposmic patients scored just one error above their threshold for age, indicating a very mild dysfunction. Because we could not test subjects of the recovered group in the acute phase of the disease, we can only report subjective information about their

Table 3 Distribution of hospitalized patients based on subjective perception of olfactory function and olfactory test result at the time of olfactory testing

\begin{tabular}{llll}
\hline & Subjective normosmia, $n(\%)$ & Subjective hyposmia, $n(\%)$ & Total, $n(\%)$ \\
\hline Olfactory test result: normosmia, $n(\%)$ & $9(36 \%)$ & $0(0 \%)$ & $9(36 \%)$ \\
Olfactory test result: hyposmia, $n(\%)$ & $13(52 \%)$ & $3(12 \%)$ & $16(64 \%)$ \\
\hline
\end{tabular}

$n$, number of patients 
Table 4 Distribution of recovered patients based on subjective perception of olfactory function and olfactory test result at the time of olfactory testing

\begin{tabular}{llll}
\hline & Subjective normosmia, $n(\%)$ & Subjective hyposmia, $n(\%)$ & Total, $n(\%)$ \\
\hline Olfactory test result: normosmia, $n(\%)$ & $20(77 \%)$ & $1(4 \%)$ & $21(81 \%)$ \\
Olfactory test result: hyposmia, $n(\%)$ & $2(8 \%)$ & $3(11 \%)$ & $5(19 \%)$ \\
\hline
\end{tabular}

$n$, number of patients

initial olfactory dysfunction. However, it should be highlighted that the proportion of recovered subjects who reported olfactory dysfunction during acute illness $(58 \%)$ is very similar to the proportion of hyposmic hospitalized patients as measured with olfactory testing (64\%). Since the congruence between subjective symptoms and performance at olfactory testing seems to be high in the "recovered" group, the proportion of these subjects reporting olfactory dysfunction during the acute phase could be considered as reliable. One study showed low agreement between self-reported olfactory function and olfactory testing [18]. However, patients were tested a mean of 18 days after the onset of olfactory dysfunction and psychophysical testing was carried out with a suprathreshold identification test, therefore some patients reporting subjective olfactory dysfunction might have already recovered or might have had an isolated olfactory threshold disorder. In our study, in order to accurately collect each participant's olfactory history and nasal symptoms during acute illness and at the time of olfactory testing, we used a structured questionnaire and the total nasal symptom score.

Overall, our findings extend current knowledge on COVID19 olfactory dysfunction by providing evidence that olfactory dysfunction can still be present in a non-negligible fraction of patients 4 months after symptoms onset and may therefore be regarded as a long-term sequela of the disease. Furthermore, we documented a highly variable subjective recovery time, from 9 days to more than 3 months. Subjective patient history and psychophysical test performed in this study suggest that some patients may need even longer time and their possibility of full recovery is currently unknown. Indeed, the pathophysiology of COVID-19-associated olfactory dysfunction is still being investigated. Evidence from autoptic series has shown a high degree of inflammation, astrogliosis, and microgliosis in the olfactory bulb of deceased COVID-19 patients [19, 20], while MRI studies have shown an early, reversible, obstruction of the olfactory cleft as a possible short-term cause of olfactory dysfunction [21, 22]. One study has pointed out that olfactory dysfunction may derive from a viral insult to olfactory neurons supporting cells [23], although an autopsy-based investigation found viral RNA in the olfactory mucosa just beneath the olfactory cleft as well as in neuroanatomical structures receiving olfactory projections, i.e., the olfactory bulb [24]. Finally, a recent study has shown significant histological recovery of olfactory structures in a COVID-19 animal model about 3 weeks after infection [25].

Several limitations must be acknowledged. This study was cross-sectional, therefore we were unable to report prospective results on patients who were "objectively" hyposmic during the acute phase of the disease. However, careful histories of olfactory dysfunction were collected in both groups included in our study. Psychophysical olfactory testing was performed with a suprathreshold identification test, therefore we were unable to test other olfactory characteristics, i.e., olfactory threshold. Although we collected the total nasal symptom score at the time of olfactory testing, rhinoscopy was not performed in this study, therefore we cannot rule out silent rhinosinusal diseases in some subjects as a cause for olfactory dysfunction; especially in the recovered group, however, all subjects who experienced olfactory loss reported a sudden and profound dysfunction, thus rendering unlikely etiological hypotheses other than SARS-CoV-2 insult. The two cohorts included different profiles of COVID-19 patients: hospitalized patients represent a moderate-to-severe spectrum of the disease; recovered subjects are more heterogenous, including five asymptomatic subjects and two subjects who had been hospitalized during the acute phase of the disease for respiratory distress; overall, recovered subjects represent a population with a milder COVID-19 phenotype. Furthermore, hospitalized patients were tested in an acute setting, which might have increased the intrinsic difficulty of the test; however, inclusion and exclusion criteria were designed in order to exclude patients with a history of neurological disease (especially dementia and parkinsonism) and/or those unable to breathe without supplementary oxygen for at least 30 minutes. All olfactory tests were conducted by physicians with specific training for IOIT administration who verified patients' fitness to adequately undergo olfactory testing. Although subjects in the recovered group were randomly selected among employees that had a positive antibody COVID-19 assay, it is possible that subjects with previous or current olfactory dysfunctions were more willing to participate. Therefore, the proportion of hyposmic subjects in the recovered group, coupled with the relatively small sample size, may be an overestimation and must be interpreted with caution. Previous population studies have reported a prevalence of olfactory dysfunction in about $20 \%$ of the general population when olfactory psychophysical testing was carried out with suprathreshold tests [26, 27]. In a series from two of the authors (C.M., S.S.) in which more than 1000 Italian healthy volunteers were screened with the same psychophysical olfactory test, around $12 \%$ had an abnormal test score and was thus hyposmic (unpublished data). Therefore, the frequency of hyposmia in the recovered group, which includes many subjects with previous COVID-19-related olfactory symptoms, seems to be slightly 
higher to that of the general population. Finally, evidence of prior SARS-CoV-2 infection was detected through either one of two commercial antibody assays with specificities of $98.5 \%$ and $99.8 \%$. Although possible, it is unlikely to have included false positive subjects in the recovered group.

\section{Conclusion}

In this study we used a standardized, 33-item psychophysical olfactory identification test tailored for the Italian population in order to investigate COVID-19-related olfactory dysfunction in 51 subjects among acute hospitalized patients and recovered subjects. Moderate-to-severely ill COVID-19 patients showed low awareness and a high frequency of olfactory dysfunction (64\% of patients). Interestingly, the proportion of recovered subjects who reported olfactory dysfunction during acute illness (58\%) was very similar to the proportion of hyposmic hospitalized patients as measured with olfactory testing (64\%). Recovered subjects showed good self-awareness of olfactory dysfunction and variable time, from days to months, to complete recovery; four of 15 (27\%) recovered subjects who reported olfactory loss during acute COVID-19 still had an abnormal test score on psychophysical testing, indicating that olfactory dysfunction may be among the long-term sequelae of COVID-19. Large prospective studies based on threshold, discrimination, and identification psychophysical olfactory tests are needed to elucidate the clinical characteristics and trajectory of olfactory dysfunction in COVID-19.

\begin{abstract}
Abbreviations COVID-19, Coronavirus disease 2019; NAAT, Nucleic acid amplification test; SARS-CoV-2, Severe acute respiratory syndrome coronavirus 2; TNSS, Total nasal symptom score
\end{abstract}

Supplementary Information The online version contains supplementary material available at https://doi.org/10.1007/s10072-021-05200-7.

Acknowledgements We thank all the COVID team of the Istituto Auxologico Italiano who worked strenuously to provide the best care to patients during this troubling time.

This project was supported by the Italian Ministry of Health (Istituto Auxologico Italiano IRCCS - Ricerca corrente, project SmellCOVID19).

Author contribution Study concept and design: JP, CM, VS, NT. Data analysis organization: JP, CM, SS, VS, NT. Data collection: JP, NT. Statistical analysis: JP, SS. Drafting of the manuscript: JP, VS, NT. Critical Revision of the manuscript: CM, SS, VS, NT.

Funding Open access funding provided by Università degli Studi di Milano within the CRUI-CARE Agreement.

Availability of data and material Data used in the preparation of this manuscript is available upon request.

\section{Declarations}

Ethical approval The protocol (23C022) was approved by the Ethics Committee of Istituto Auxologico Italiano.

Consent to participate Due to the exceptional emergency situation, informed consent was obtained verbally and recorded by the clinician who conducted the olfactory test (J.P., N.T.). The study conforms with World Medical Association Declaration of Helsinki.

Conflict of Interest JP, SS, and NT have no relevant financial or nonfinancial interests to disclose.

$\mathrm{CM}$ is the owner of the patent of the Italian Olfactory Identification Test (IOIT) (N.0001383361, issued by the Italian Ministry of Economic Development). The patent has not been licensed to third parties and the author has not received royalties linked to this patent.

VS is in the Editorial Board of Amyotrophic Lateral Sclerosis, European Neurology, American Journal of Neurodegenerative Diseases, and Frontiers in Neurology; received compensation for consulting services and/or speaking activities from AveXis, Cytokinetics, Italfarmaco, and Zambon; and receives or has received research supports from the Italian Ministry of Health (Grant RF-201302355764), Fondazione Italiana di Ricerca per la SLA-AriSLA (Grant Exomefals and Novals), Fondazione Regionale per la Ricerca Biomedica Regione Lombardia (Project no. 2015-0023), and E-RARE JTC (Project Repetomics).

Open Access This article is licensed under a Creative Commons Attribution 4.0 International License, which permits use, sharing, adaptation, distribution and reproduction in any medium or format, as long as you give appropriate credit to the original author(s) and the source, provide a link to the Creative Commons licence, and indicate if changes were made. The images or other third party material in this article are included in the article's Creative Commons licence, unless indicated otherwise in a credit line to the material. If material is not included in the article's Creative Commons licence and your intended use is not permitted by statutory regulation or exceeds the permitted use, you will need to obtain permission directly from the copyright holder. To view a copy of this licence, visit http://creativecommons.org/licenses/by/4.0/.

\section{References}

1. Mao L, Jin H, Wang M, Hu Y, Chen S, He Q, Chang J, Hong C, Zhou Y, Wang D, Miao X, Li Y, Hu B (2020) Neurologic manifestations of hospitalized patients with coronavirus disease 2019 in Wuhan, China. JAMA Neurol 77:683-690. https://doi.org/10. 1001/jamaneurol.2020.1127

2. Spinato G, Fabbris C, Polesel J, Cazzador D, Borsetto D, Hopkins C, Boscolo-Rizzo P (2020) Alterations in smell or taste in mildly symptomatic outpatients with SARS-CoV-2 infection. JAMA. 323: 2089-2090. https://doi.org/10.1001/jama.2020.6771

3. Giacomelli A, Pezzati L, Conti F et al (2020) Self-reported olfactory and taste disorders in SARS-CoV-2 patients: a cross-sectional study. Clin Infect Dis:1-2. https://doi.org/10.1093/cid/ciaa330

4. Lechien JR, Chiesa-Estomba CM, De Siati DR et al (2020) Olfactory and gustatory dysfunctions as a clinical presentation of mild-to-moderate forms of the coronavirus disease (COVID-19): a multicenter European study. Eur Arch Otorhinolaryngol 277:22512261. https://doi.org/10.1007/s00405-020-05965-1 
5. Bénézit F, Le Turnier P, Declerck C et al (2020) Utility of hyposmia and hypogeusia for the diagnosis of COVID-19. Lancet Infect Dis 3099:19-20. https://doi.org/10.1016/S1473-3099(20)30297-8

6. Moein ST, Hashemian SMR, Mansourafshar B, Khorram-Tousi A, Tabarsi P, Doty RL (2020) Smell dysfunction: a biomarker for COVID-19. Int Forum Allergy Rhinol 10:944-950. https://doi. org/10.1002/alr.22587

7. Tsivgoulis G, Fragkou PC, Delides A, Karofylakis E, Dimopoulou D, Sfikakis PP, Tsiodras S (2020) Quantitative evaluation of olfactory dysfunction in hospitalized patients with coronavirus [2] (COVID-19). J Neurol 267:2193-2195. https://doi.org/10.1007/ s00415-020-09935-9

8. Hornuss D, Lange B, Schröter N Rieg S, Kern WV, Wagner D (2020) Anosmia in COVID-19 patients. Clin Microbiol Infect. 26(10):1426-1427. https://doi.org/10.1016/j.cmi.2020.05.017

9. Petrocelli M, Ruggiero F, Baietti AM, Pandolfi P, Salzano G, Salzano FA, Lechien JR, Saussez S, de Riu G, Vaira LA (2020) Remote psychophysical evaluation of olfactory and gustatory functions in early-stage coronavirus disease 2019 patients: the Bologna experience of 300 cases. J Laryngol Otol 134:571-576. https://doi. org/10.1017/S0022215120001358

10. Vaira LA, Deiana G, Fois AG, Pirina P, Madeddu G, de Vito A, Babudieri S, Petrocelli M, Serra A, Bussu F, Ligas E, Salzano G, de Riu G (2020) Objective evaluation of anosmia and ageusia in COVID-19 patients: single-center experience on 72 cases. Head Neck 42:1252-1258. https://doi.org/10.1002/hed.26204

11. Lechien JR, Ducarme M, Place S, Chiesa-Estomba CM, Khalife M, de Riu G, Vaira LA, de Terwangne C, Machayekhi S, Marchant A, Journe F, Saussez S (2020) Objective olfactory findings in hospitalized severe COVID-19 patients. Pathogens 9:627. https://doi.org/ $10.3390 /$ pathogens 9080627

12. Moein ST, Hashemian SMR, Tabarsi P, Doty RL (2020) Prevalence and reversibility of smell dysfunction measured psychophysically in a cohort of COVID-19 patients. Int Forum Allergy Rhinol:1-9. https://doi.org/10.1002/alr.22680

13. Vaira LA, Hopkins C, Petrocelli M, Lechien JR, Soma D, Giovanditto F, Rizzo D, Salzano G, Piombino P, Saussez S, de Riu G (2020) Do olfactory and gustatory psychophysical scores have prognostic value in COVID-19 patients? A prospective study of 106 patients. J Otolaryngol Head Neck Surg 49:56. https://doi. org/10.1186/s40463-020-00449-y

14. Le Bon SD, Pisarski N, Verbeke J et al (2020) Psychophysical evaluation of chemosensory functions 5 weeks after olfactory loss due to COVID-19: a prospective cohort study on 72 patients. Eur Arch Otorhinolaryngol 278:101-108. https://doi.org/10.1007/ s00405-020-06267-2

15. Otte MS, Eckel HNC, Poluschkin L, Klussmann JP, Luers JC (2020) Olfactory dysfunction in patients after recovering from COVID-19. Acta Otolaryngol 140:1-4. https://doi.org/10.1080/ 00016489.2020.1811999

16. Maremmani C, Rossi G, Tambasco N, Fattori B, Pieroni A, Ramat S, Napolitano A, Vanni P, Serra P, Piersanti P, Zanetti M, Coltelli M, Orsini M, Marconi R, Purcaro C, Rossi A, Calabresi P, Meco G (2012) The validity and reliability of the Italian Olfactory Identification Test (IOIT) in healthy subjects and in Parkinson's disease patients. Parkinsonism Relat Disord 18:788-793. https:// doi.org/10.1016/j.parkreldis.2012.03.021

17. Downie SR, Andersson M, Rimmer J, Leuppi JD, Xuan W, Akerlund A, Peat JK, Salome CM (2004) Symptoms of persistent allergic rhinitis during a full calendar year in house dust mitesensitive subjects. Allergy 59:406-414. https://doi.org/10.1111/j. 1398-9995.2003.00420.x

18. Lechien JR, Cabaraux P, Chiesa-Estomba CM, Khalife M, Hans S, Calvo-Henriquez C, Martiny D, Journe F, Sowerby L, Saussez S (2020) Objective olfactory evaluation of self-reported loss of smell in a case series of 86 COVID-19 patients. Head Neck 42:15831590. https://doi.org/10.1002/hed.26279

19. Matschke J, Lütgehetmann M, Hagel C, Sperhake JP, Schröder AS, Edler C, Mushumba H, Fitzek A, Allweiss L, Dandri M, Dottermusch M, Heinemann A, Pfefferle S, Schwabenland M, Sumner Magruder D, Bonn S, Prinz M, Gerloff C, Püschel K, Krasemann S, Aepfelbacher M, Glatzel M (2020) Neuropathology of patients with COVID-19 in Germany: a postmortem case series. Lancet Neurol 19:919-929. https://doi.org/10. 1016/s1474-4422(20)30308-2

20. Schurink B, Roos E, Radonic T, Barbe E, Bouman CSC, de Boer HH, de Bree GJ, Bulle EB, Aronica EM, Florquin S, Fronczek J, Heunks LMA, de Jong MD, Guo L, du Long R, Lutter R, Molenaar PCG, Neefjes-Borst EA, Niessen HWM, van Noesel CJM, Roelofs JJTH, Snijder EJ, Soer EC, Verheij J, Vlaar APJ, Vos W, van der Wel NN, van der Wal AC, van der Valk P, Bugiani M (2020) Viral presence and immunopathology in patients with lethal COVID-19: a prospective autopsy cohort study. Lancet Microbe 1:e290-e299. https://doi.org/10.1016/s2666-5247(20)30144-0

21. Eliezer M, Hamel A-L, Houdart E, Herman P, Housset J, Jourdaine C, Eloit C, Verillaud B, Hautefort C (2020) Loss of smell in COVID-19 patients: MRI data reveals a transient edema of the olfactory clefts. Neurology 95:e3145-e3152. https://doi.org/10. 1212/WNL.0000000000010806

22. Niesen M, Trotta N, Noel A, Coolen T, Fayad G, Leurkin-Sterk G, Delpierre I, Henrard S, Sadeghi N, Goffard JC, Goldman S, de Tiège X (2021) Structural and metabolic brain abnormalities in COVID-19 patients with sudden loss of smell. Eur J Nucl Med Mol Imaging. https://doi.org/10.1007/s00259-020-05154-6

23. Brann DH, Tsukahara T, Weinreb C, Lipovsek M, van den Berge K, Gong B, Chance R, Macaulay IC, Chou HJ, Fletcher RB, Das D, Street K, de Bezieux HR, Choi YG, Risso D, Dudoit S, Purdom E, Mill J, Hachem RA, Matsunami H, Logan DW, Goldstein BJ, Grubb MS, Ngai J, Datta SR (2020) Non-neuronal expression of SARS-CoV-2 entry genes in the olfactory system suggests mechanisms underlying COVID-19-associated anosmia. Sci Adv 6:1-20. https://doi.org/10.1126/sciadv.abc5801

24. Meinhardt J, Radke J, Dittmayer C, Franz J, Thomas C, Mothes R, Laue M, Schneider J, Brünink S, Greuel S, Lehmann M, Hassan O, Aschman T, Schumann E, Chua RL, Conrad C, Eils R, Stenzel W, Windgassen M, Rößler L, Goebel HH, Gelderblom HR, Martin H, Nitsche A, Schulz-Schaeffer WJ, Hakroush S, Winkler MS, Tampe B, Scheibe F, Körtvélyessy P, Reinhold D, Siegmund B, Kühl AA, Elezkurtaj S, Horst D, Oesterhelweg L, Tsokos M, Ingold-Heppner B, Stadelmann C, Drosten C, Corman VM, Radbruch H, Heppner FL (2020) Olfactory transmucosal SARS-CoV-2 invasion as a port of central nervous system entry in individuals with COVID-19. Nat Neurosci 24:168-175. https://doi.org/10.1038/s41593-020-00758-5

25. Urata S, Maruyama J, Kishimoto-Urata M et al (2021) Regeneration profiles of olfactory epithelium after SARS-CoV-2 infection in golden Syrian hamsters. ACS Chem Neurosci:acschemneuro.0c00649. https://doi.org/10.1021/acschemneuro.0c00649

26. Brämerson A, Johansson L, Ek L et al (2004) Prevalence of olfactory dysfunction: the Skövde population-based study. Laryngoscope 114:733-737. https://doi.org/10.1097/00005537200404000-00026

27. Murphy C, Schubert CR, Cruickshanks KJ, Klein BE, Klein R, Nondahl DM (2002) Prevalence of olfactory impairment in older adults. J Am Med Assoc 288:2307-2312. https://doi.org/10.1001/ jama.288.18.2307

Publisher's note Springer Nature remains neutral with regard to jurisdictional claims in published maps and institutional affiliations. 